\title{
New challenges from Covid-19 pandemic: an unexpected opportunity to enlighten the link between viral infections and brain disorders?
}

\author{
Alessandro Gialluisi ${ }^{1}$ (D) - Giovanni de Gaetano ${ }^{1}$ • Licia lacoviello ${ }^{1,2}$ \\ Received: 7 April 2020 / Accepted: 25 April 2020 / Published online: 6 May 2020 \\ (C) Fondazione Società Italiana di Neurologia 2020
}

Dear Editor,

We read with great interest recent reports of sensorial impairments in Covid-19 patients, both in the early [1] and in a later phase of the infection [2]. It is time for the scientific community to consider potential challenges and opportunities of the Covid-19 pandemic beyond clinical emergency, based on these and other observations. Among these, neurological consequences of the disease may represent an unexpected feature of translational relevance.

Indeed, infectious diseases have been associated with an increased risk of neurodegenerative and neuropsychiatric disorders $[3,4]$. While this link may look obvious for multiple sclerosis, due to its auto-immune nature [5], it is less so for disorders due to the accumulation of neurotoxic aggregates, like Parkinson's and Alzheimer's disease. Influenza epidemics are associated with neurological manifestations, and the $\mathrm{H}_{5} \mathrm{~N}_{1}$ virus reportedly induces Parkinsonian pathology in mice, both findings possibly explained by the activation of inflammatory pathways [4]. However, the exact mechanisms of these effects and whether coronaviruses show a similar action remain unclear. The current Covid-19 pandemic provides an unprecedented opportunity to disentangle the link between viral/ infectious diseases, inflammation, and the risk of brain disorders, as mentioned here below.

Many Covid-19 patients present with loss of taste and olfaction [1], even after the acute phase of infection [2]. Hyposmia is a common symptom of different neurodegenerative and

Alessandro Gialluisi

alessandro.gialluisi@gmail.com

1 Department of Epidemiology and Prevention, IRCCS NEUROMED, Via dell'Elettronica, 86077 Pozzilli, Italy

2 Research Center in Epidemiology and Preventive Medicine (EPIMED), Department of Medicine and Surgery, University of Insubria, Varese, Italy neuropsychiatric disorders [6] and has been associated with levels of interleukin-6 (IL-6), an inflammatory biomarker deeply investigated in brain disorders [7]. Of note, IL-6 action is blocked by tocilizumab, a drug currently used to inhibit cytokine storm in Covid-19 patients [8]. A recent study revealed creactive protein levels above the low-grade inflammation threshold (>3 mg/l) for three out of four patients presenting with loss of taste and olfaction, which were reported to be more intense and long-lasting than those in common cold disease [2]. Therefore, accumulating evidence suggests systemic inflammation may be a potential pathway to explain neurological sequelae of Covid-19, a hypothesis which should be investigated more in depth.

About half of hospitalized patients are $>55$ years [9]: the resulting higher age-related risk of neurodegenerative disorders is a good setting to investigate triggering and double-hit mechanisms previously hypothesized for viral infections [4]. The high diffusion of Covid-19 (1,873,265 ascertained cases worldwide as of April 14, 2020; https://www.ecdc.europa.eu/ en/geographical-distribution-2019-ncov-cases) ensures a high power to detect effects on the incident risk of brain disorders. Indeed, the rapid spread of the infection through different ethnicities and cultural and genetic backgrounds may allow planning robust multicenter studies.

To sum up, the current Covid-19 pandemic provides an extraordinary window to implement a worldwide collaborative effort, aimed at enlightening the largely unexplored relationship between viral infections, inflammation, and brain disorders. To this purpose, coordination of hospitals and general practice and public health institutions will be needed to collect, store, and allow access to electronic health records during and after the epidemic. We "call to arms" clinicians and researchers worldwide, to take an unexpected advantage from a painful event and make it an extraordinary opportunity to fight brain disorders, which represent a huge challenge and a heavy burden for national health systems. 
Funding information AG was supported by Fondazione Umberto Veronesi.

\section{Compliance with ethical standards}

Conflict of interest The authors declare that they have no conflict of interest.

\section{References}

1. Giacomelli A, Laura P, Conti F et al (2020) Self-reported olfactory and taste disorders in SARS-CoV-2 patients: a cross-sectional study | clinical infectious diseases | Oxford Academic. Clin Infect Dis. https://doi.org/10.1093/cid/ciaa330

2. Wölfel R, Corman VM, Guggemos W et al (2020) Virological assessment of hospitalized patients with COVID-2019. Nature:1-10. https://doi.org/10.1038/s41586-020-2196-x

3. Bullido MJ, Carter C, Clerici M, et al (2017) Microbes and Alzheimer's disease 51:979-984. https://doi.org/10.3233/JAD160152.Microbes
4. Henry J, Smeyne RJ, Jang H, Miller B, Okun MS (2010) Parkinsonism and neurological manifestations of influenza throughout the 20th and 21st centuries. Parkinsonism Relat Disord 16:566571. https://doi.org/10.1016/j.parkreldis.2010.06.012

5. Fierz W (2017) Multiple sclerosis: an example of pathogenic viral interaction? Virol J 14:42

6. Turetsky BI, Hahn CG, Borgmann-Winter K, Moberg PJ (2009) Scents and nonsense: olfactory dysfunction in schizophrenia. Schizophr Bull 35:1117-1131. https://doi.org/10.1093/schbul/ sbp111

7. Henkin RI, Schmidt L, Velicu I (2013) Interleukin 6 in hyposmia. JAMA Otolaryngol Neck Surg 139:728-734. https://doi.org/10. 1001/jamaoto.2013.3392

8. Mehta P, McAuley DF, Brown M et al (2020) COVID-19: consider cytokine storm syndromes and immunosuppression. Lancet. 395: 1033-1034. https://doi.org/10.1016/S0140-6736(20)30628-0

9. Bialek S, Boundy E, Bowen V et al (2020) Severe outcomes among patients with coronavirus disease 2019 (COVID-19) - United States, February 12-March 16, 2020. MMWR Morb Mortal Wkly Rep 69:343-346. https://doi.org/10.15585/mmwr.mm6912e2

Publisher's note Springer Nature remains neutral with regard to jurisdictional claims in published maps and institutional affiliations. 